\title{
BIO-NSPPIRED EMERGENT CONTROL OF LOCOMOTION SYSTEMS
}




\section{WORLD SCIENTIFIC SERIES ON NONLINEAR SCIENCE}

\section{Editor: Leon O. Chua}

University of California, Berkeley

\section{Series A. MONOGRAPHS AND TREATISES}

Volume 29: Nonlinear \& Parametric Phenomena*

V. Damgov

Volume 30: Quasi-Conservative Systems: Cycles, Resonances and Chaos

A. D. Morozov

Volume 31: CNN: A Paradigm for Complexity

L. O. Chua

Volume 32: From Order to Chaos II

L. P. Kadanoff

Volume 33: Lectures in Synergetics

V. I. Sugakov

Volume 34: Introduction to Nonlinear Dynamics*

L. Kocarev \& M. P. Kennedy

Volume 35: Introduction to Control of Oscillations and Chaos

A. L. Fradkov \& A. Yu. Pogromsky

Volume 36: Chaotic Mechanics in Systems with Impacts \& Friction

B. Blazejczyk-Okolewska, K. Czolczynski, T. Kapitaniak \& J. Wojewoda

Volume 37: Invariant Sets for Windows - Resonance Structures, Attractors, Fractals and Patterns

A. D. Morozov, T. N. Dragunov, S. A. Boykova \& O. V. Malysheva

Volume 38: Nonlinear Noninteger Order Circuits \& Systems - An Introduction P. Arena, R. Caponetto, L. Fortuna \& D. Porto

Volume 39: The Chaos Avant-Garde: Memories of the Early Days of Chaos Theory Edited by Ralph Abraham \& Yoshisuke Ueda

Volume 40: Advanced Topics in Nonlinear Control Systems Edited by T. P. Leung \& H. S. Qin

Volume 41: Synchronization in Coupled Chaotic Circuits and Systems C. W. Wu

Volume 42: Chaotic Synchronization: Applications to Living Systems E. Mosekilde, Y. Maistrenko \& D. Postnov

Volume 43: Universality and Emergent Computation in Cellular Neural Networks R. Dogaru

Volume 44: Bifurcations and Chaos in Piecewise-Smooth Dynamical Systems Z. T. Zhusubaliyev \& E. Mosekilde

Volume 45: Bifurcation and Chaos in Nonsmooth Mechanical Systems J. Awrejcewicz \& C.-H. Lamarque

Volume 46: Chaos, Bifurcations and Fractals Around Us W. Szemplińska-Stupnicka 
Series Editor: Leon O. Chua

\section{BIO-NSSPIRED EMERGENT CONTAOL OF LOCOMOTION SYSTEMS}

\section{Mattia Frasca \\ Paolo Arena \\ Luigi Fortuna}

Università degli Studi di Catania, Italy 
Published by

World Scientific Publishing Co. Pte. Ltd.

5 Toh Tuck Link, Singapore 596224

USA office: Suite 202, 1060 Main Street, River Edge, NI 07661

UK office: 57 Shelton Street, Covent Garden, London WC2H 9HE

British Library Cataloguing-in-Publication Data

A catalogue record for this book is available from the British Library.

\section{BIO-INSPIRED EMERGENT CONTROL OF LOCOMOTION SYSTEMS}

Copyright $\odot 2004$ by World Scientific Publishing Co. Pte. Ltd.

All rights reserved. This book, or parts thereof, may not be reproduced in any form or by any means, electronic or mechanical, including photocopying, recording or any information storage and retrieval system now known or to be invented, without written permission from the Publisher.

For photocopying of material in this volume, please pay a copying fee through the Copyright Clearance Center, Inc., 222 Rosewood Drive, Danvers, MA 01923, USA. In this case permission to photocopy is not required from the publisher.

ISBN 981-238-919-9

Printed in Singapore by Mainland Press 
To our parents 
This page intentionally left blank 


\section{Preface}

Living creatures show distinct abilities to interact adaptively with their environment. These characteristics find their roots in the self-organizing dynamics of neural circuits, which in nonlinear science represent the highest example of emergent system behavior. In this work bio-inspired locomotion control is achieved by means of arrays of locally coupled nonlinear systems (Cellular Nonlinear Networks). The main point of the approach is that nonlinear oscillators are an efficient way not only to model the principles underlying neural structures devoted to locomotion control in living creatures, but more importantly to implement them to build autonomous walking robots. In this context the concepts of self-organization and synchronization, universal paradigms of science and real world laws, play a fundamental role in the emergence of the complex behavior of the network of nonlinear circuits. The proposed method is efficient at all levels of the hierarchical control of locomotion: the low level of gait generation, reflex and sensory feedback implementation, and the high level of posture stabilization and behavioral modules. The strategy introduced is hardwareoriented, so the concepts of emergent behavior and self-organization can be appreciated in chip devices applied to walking machines. Experimental results obtained with a prototype of the VLSI chip implementing the Cellular Nonlinear Network generating the locomotion pattern and tests on a bio-inspired insect-like robot are presented.

This book constitutes a complete monograph on new locomotion control strategies of bio-inspired robots implemented through Cellular Nonlinear Networks (CNNs). The new approach for locomotion control, based on emergent properties of nonlinear dynamical systems, is exhaustively described and several case studies are presented. Topics related to the problem of locomotion control from low level to high level control are addressed. 
The book contains both a detailed introduction on the fundamentals of Cellular Nonlinear Networks and bio-inspired control and research results with particular emphasis on applications. The proposed methodology is illustrated with the help of several examples dealing with bio-inspired hexapod robots. The present book is thus aimed both at newcomers to the field of bio-inspired locomotion control and graduate students in advanced courses on bio-inspired robotics as well as $\mathrm{PhD}$ students and researches both from academic institutions and industrial companies. In particular, the main features of the approach beneficial to the reader are the following: analog computation is used to control locomotion systems; realization of well-tested circuit for control is discussed; each reader could implement its own circuits at low cost (few dollars for each analog unit).

We wish to express our gratitude to Prof. L. O. Chua who has been a constant source of inspiration and encouragement for this work. Part of this work has been partially supported by Office of Naval Research, project number N00014-03-1-0392, and by MIUR ("Ministero dell'Istruzione, dell'Universitá e della Ricerca") under project PRIN "Innovative Bio-Inspired Strategies for the Control of Motion Systems". Moreover, we thank STMicroelectronics (Catania site) for its fruitful support. 


\section{Contents}

Preface vii

1. Introduction 1

1.1 The Central Pattern Generator (CPG) . . . . . . . . . . . 2

1.2 Locomotion control in hexapods . . . . . . . . . . 4

1.3 Main topics of the work . . . . . . . . . . . 6

2. CNN-based Central Pattern Generators 9

2.1 Introduction . . . . . . . . . . . . . . . 9

2.2 Brief overview on CNN architectures . . . . . . . . 12

2.3 The $\mathrm{CPG}$ neuron . . . . . . . . . . . . . . 14

2.3 .1 The synapse model . . . . . . . . . . . . . 18

2.4 The CNN-based CPG . . . . . . . . . . . . . 21

2.4.1 RD-CNNs to design artificial locomotion patterns . . 22

2.4.2 Guidelines for CNN-based CPG design . . . . . . . 24

2.5 Example: the caterpillar gait for hexapods . . . . . . . . 28

2.6 A spatio-temporal algorithm for controlling locomotion of a hexapod robot . . . . . . . . . . . 32

2.7 Motor-neurons and inter-neurons $\ldots \ldots \ldots . \ldots 37$

3. CNN-based CPGs with sensory feedback and VLSI implementation

3.1 Direction control . . . . . . . . . . . . . . . . . 43

3.1 .1 The CPG cell . . . . . . . . . . . . . . . . 44

3.1.2 CPG with sensory feedback for direction control . . . 47

3.2 Feedback from ground contact sensors . . . . . . . . 50 
3.2.1 Behavior of the CNN neuron driven by a periodic forcing signal ..................... 51

3.2.2 CPG with ground contact feedback: results . . . . 54

3.3 Reflex implementation . . . . . . . . . . . . 57

3.3 .1 The elevator reflex . . . . . . . . . . . 57

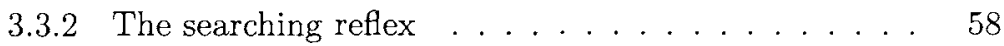

3.4 Speed control . . . . . . . . . . . . . . . 60

3.4 .1 Speed of the gait . . . . . . . . . . 61

3.4 .2 Locomotion pattern . . . . . . . . . . . . . 63

3.5 The CNN-based CPG VLSI chip . . . . . . . . . 63

3.5 .1 The hybrid approach . . . . . . . . . . 63

3.5 .2 The VLSI Circuit Design . . . . . . . . . . . . . 64

3.5 .3 Experimental results ............. 66

4. Decentralized locomotion control $\quad 73$

4.1 CNN-based decentralized control model . . . . . . . . 73

4.1.1 The decentralized control paradigm . . . . . . . . 75

4.1.2 The CNN leg controller . . . . . . . . . . . 77

4.1.3 The whole control system and results . . . . . . . 80

4.1.3.1 CNN Decentralized Control ... . . . . . 80

4.1.3.2 Choice of the parameters of the model . . . 83

4.1.3.3 Robustness of the CNN Decentralized Controller ............... 86

4.2 Integrate-and-fire neurons and decentralized control . . . 88

4.2 .1 The leg controller . . . . . . . . . . . . . 90

4.2.1.1 Biological motivations . . . . . . . . . 90

4.2.1.2 The integrate-and-fire neuron . . . . . . . 90

4.2.1.3 Scheme of the leg controller . . . . . . . 91

4.2.1.4 The elevator reflex . . . . . . . . . 9 95

4.2.2 The whole control scheme . . . . . . . . . 97

4.3 CPG and decentralized control . . . . . . . . . . . . 99

5. A gallery of bio-inspired robots 101

5.1 Lampbot: A lamprey robot controlled by RD-CNN . . . . 101

5.2 MTA hexbot: a hexapod robot controlled by MTA-CNN . . 106

5.3 MTA hexbot II: a remote-controlled hexapod robot . . . . 110

5.4 MTA hexbot III: a robot driven by the CNN-based CPG VLSI chip . . . . . . . . . . . . . . . . 112 
6. High-level analog control: attitude control and Motor Maps

6.1 CNN-based attitude control . . . . . . . . . . . . 115

6.1.1 The CNN for gait control . . . . . . . . . . 117

6.1 .2 The attitude control CNN . . . . . . . . . . . . 119

6.1 .3 Experimental tests . . . . . . . . . . . . . . 123

6.2 Motor Maps and attitude control . . . . . . . . . . 125

6.2 .1 Motor Maps . . . . . . . . . . . . . . . . . . . 125

6.2 .2 Motor Maps for Chaos Control . . . . . . . . . . . 129

6.2.3 Motor Maps for attitude control . . . . . . . . . . . 132

6.2.4 Motor Map-based attitude control in a simplified biped model . . . . . . . . . . . . . . 136

6.3 Learning with Motor Maps . . . . . . . . . . . . . 142

7. High-level analog control: Turing patterns and autowaves 145

7.1 Reaction-Diffusion CNN . . . . . . . . . . . . 146

7.2 Navigation control based on Turing patterns . . . . . . 148

7.2 .1 Turing patterns and CNNs . . . . . . . . . 149

7.2.2 From CNN patterns to action patterns . . . . . . 151

7.2 .3 Experimental Setup . . . . . . . . . . . . . . . 153

7.2 .4 To probe further . . . . . . . . . . . . . . . 154

7.3 Navigation control based on autowaves . . . . . . . . 156

7.3.1 The CNN algorithm . . . . . . . . . . 157

7.3.2 Implementation on a roving robot and experimental results . . . . . . . . . . . . 160

8. Conclusions 165

Appendix A HexaDyn and CNNLab: two tools for bioinspired locomotion control

A.1 HexaDyn, a simulator for the hexapod robot . . . . . . 171

A.2 CNNLab . . . . . . . . . . . . . . . . . . 173

$\begin{array}{lll}\text { Appendix B Design of the CNN circuit } & 177\end{array}$

Appendix C A chaos-based sensor for bio-inspired robots $\quad 181$

C.1 Continuous CPPM . . . . . . . . . . . . . . . . 181

C.2 The CPPM Sonar . . . . . . . . . . . . . 183 
References

Index 